\title{
Incidental discovery of mucinous adenocarcinoma of the prostate following transurethral resection of the prostate: A report of two cases and a literature review
}

\author{
LIJIE ZHANG ${ }^{1}$, LIHUA ZHANG $^{2},{\text { MING } \text { CHEN }^{1} \text { and QUAN FANG }}^{1,3}$ \\ Departments of ${ }^{1}$ Urology and ${ }^{2}$ Pathology, Zhongda Hospital, Southeast University College of Medicine, Nanjing, \\ Jiangsu 210096; ${ }^{3}$ Department of Surgery, Shanghai East International Hospital, Shanghai 200120, P.R. China
}

Received February 10, 2018; Accepted July 26, 2018

DOI: $\quad 10.3892 / \mathrm{mco} .2018 .1686$

\begin{abstract}
Mucinous carcinoma is an unusual subtype of prostate cancer. In particular, mucinous adenocarcinomas identified following transurethral resection of the prostate (TURP) are extremely rare. The present study conducted are retrospective analysis of two cases of mucinous carcinoma of the prostate, which were incidentally diagnosed following histological examination of the specimens obtained by TURP. The pathological findings, treatment regimen and clinical course of the two cases were reviewed. One of the patients, whose surgical specimen stained positive for carcinoembryonic antigen (CEA) and negative for prostate-specific antigen (PSA), did not respond to androgen deprivation therapy (ADT). However, the other patient, whose specimen stained positive for PSA, was responsive to ADT, resulting in a better prognosis. Therefore, absence of PSA staining in surgical prostate specimens may be associated with a poor response to ADT and a worse prognosis.
\end{abstract}

\section{Introduction}

Mucinous adenocarcinoma of the prostate is a rare morphological variant of prostate cancer, which is defined by the presence of extravasated mucin in $>25 \%$ of the tumor volume $(1,2)$. Of all prostate cancer types, the incidence of this rare malignancy is $\sim 0.2 \%$ (3). The overall incidence of prostate cancer diagnosis following transurethral resection of the prostate (TURP) has sharply decreased following introduction of PSA screening (4). Mucinous carcinoma of the prostate differs from secondary prostatic involvement by mucinous adenocarcinomas arising in other sites, such as the rectum or bladder, in terms of typical

Correspondence to: Dr Quan Fang, Department of Surgery, Shanghai East International Hospital, 150 Jimo Road, Shanghai 200120, P.R. China

E-mail: quan.fang@seimc.com.cn

Key words: prostate gland, mucinous carcinoma, transurethral resection of the prostate, diagnosis, treatment morphology and cytological characteristics (5). However, the primary origin of less well-differentiated tumors may be difficult to identify. Negative staining for PSA may help differentiate between primary and secondary tumors; however, PSA staining in undifferentiated prostatic cancer may also be negative (6), and is therefore unreliable.

To the best of our knowledge, mucinous adenocarcinoma of the prostate incidentally identified in specimens obtained via TURP has not yet been reported in any previous study. The present study reported two cases of mucinous adenocarcinoma that were incidentally identified following TURP. The clinical progression of the disease, in addition to the treatment options and prognostic factors, were also reviewed.

\section{Case studies}

Case 1. A 67-year-old man was admitted to Zhongda Hospital (Nanjing, China) with edema of the bilateral lower extremities for a total of 6months in July, 2015. In the local hospital (Muyang County Hospital, Suqian, China), the patient was diagnosed with bilateral hydronephrosis and bilateral ureteral colic on urologic ultrasound examination. A pelvic computed tomography (CT) scan revealed an enlarged prostate and a focal thickening of the bladder wall. Magnetic resonance imaging examination revealed that the tumor had invaded the prostatic capsule (Fig. 1A), with possible bladder wall invasion (Fig. 1B). The results of the laboratory tests were as follows: Blood urea nitrogen, $21.7 \mathrm{mmol} / 1$; creatinine, $634 \mu \mathrm{mol} / \mathrm{l}$; carcinoembryonic antigen (CEA), $8.3 \mathrm{ng} / \mathrm{ml}$; and prostate-specific antigen (PSA), $4.2 \mathrm{mg} / \mathrm{ml}$. The patient underwent cystoscopy to evaluate the focal thickening of the bladder wall, and TURP for the enlarged prostate, in addition to an ultrasound-guided bilateral percutaneous nephrostomy to decompress the upper urinary system and relieve the hydronephrosis. On histological examination of the TURP specimen, prostatic adenocarcinoma and mucinous adenocarcinoma were identified. The biopsy of the bladder wall thickening was inconclusive. A radionuclide bone scan was negative for metastasis. Three weeks later, the patient underwent radical cystoprostatectomy with bilateral pelvic lymphadenectomy and bilateral ureterostomy. Pathological examination of the surgically obtained specimen confirmed the diagnosis of prostatic adenocarcinoma with a Gleason 

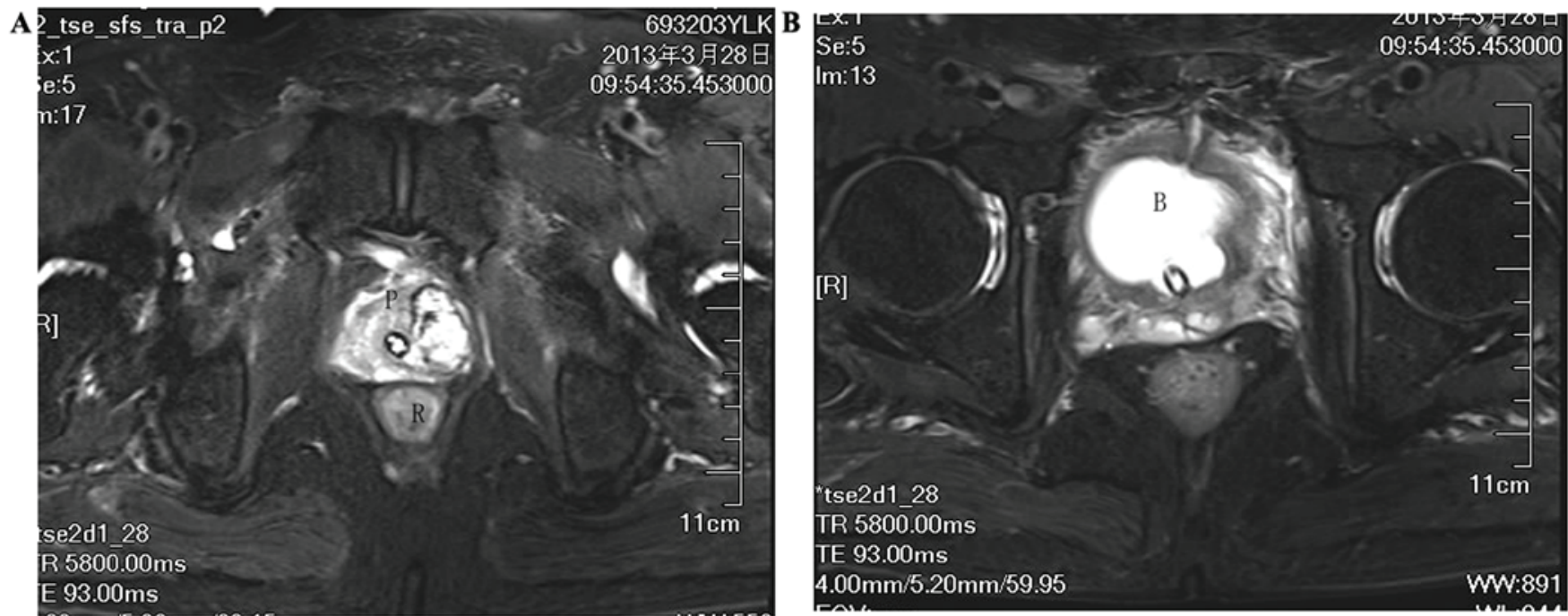

Figure 1. Magnetic resonance imaging of the prostate in Case 1. (A) A large nodule in the left lobe of the prostate is seen invading the prostatic capsule (P, prostate; R, rectum); (B) localized thickening of the bladder wall indicating possible tumor invasion (B, bladder).

score of $4+5=9 / 10$,which was the same as the Gleason score of the TURP sample. The mucinous carcinomatous component comprised $25 \%$ of the tumor. Tumor invasion was observed in the peri-prostatic adipose tissue, bilateral seminal vesicles, all layers of the bladder wall and the surgical margins of the prostate-bladder neck. All pelvic lymph nodes were negative for tumor involvement (6 lymph nodes on the left and 9 on the right side). On immunohistochemical examination, the tumor stained positive for cytokeratin (CK)20, CEA, Ki-67 (20\%) and $\beta$-catenin, and negative for CK7, androgen receptor (AR), prostate-specific membrane antigen (PSMA) and PSA (Fig. 2). Postoperatively, the patient underwent adjuvant androgen deprivation therapy (ADT) with bicalutamide $150 \mathrm{mg}$ per day and goserelin $3.6 \mathrm{mg}$ every 4 weeks for 1 year, and the total PSA level remained constant at $<0.003 \mathrm{ng} / \mathrm{ml}$ during that time. The follow-up bone scan was negative for tumor metastasis. At 1.5 years after surgery, an abdominal CT scan revealed multiple metastatic lesions in the peritoneum and a positron emission tomography (PET) scan revealed metastatic lesions to the pelvic bones and the right femur. The serum CEA levels had increased to $221.6 \mathrm{ng} / \mathrm{ml}$ and total PSA remained at $<0.003 \mathrm{ng} / \mathrm{ml}$. The serum testosterone levels were $<5 \mathrm{ng} / \mathrm{dl}$. The patient subsequently underwent localized radiotherapy for bone metastasis. The total dose of the radiotherapy was $20 \mathrm{~Gy}$ (40 Gy/fraction) and the metastatic lesions reduced in size following the treatment. Chemotherapy was not considered to be an option due to the patient's underlying congestive cardiac failure. The patient has been on palliative care with pain control. The last follow-up of the patient was in December 2017, and the patient was in a stable condition.

Case 2. A 70-year-old man who underwent TURP at a local hospital 6 weeks earlier was admitted to Zhongda Hospital in September 2014. The patient's preoperative serum PSA level was $0.95 \mathrm{ng} / \mathrm{ml}$. A pathological examination of the TURP specimen revealed mucinous adenocarcinoma of the prostate, with a Gleason score of $4+4=8 / 10$. A total of $100 \%$ of the tumor mass was mucinous carcinoma. A PET scan revealed no bone metastases. Subsequently, the patient underwent radical prostatectomy and bilateral pelvic lymphadenectomy. Histological examination of the surgical specimens revealed mucinous adenocarcinoma in the left lobe of the prostate, with a Gleason score of 8 . The surgical margins, seminal vesicles and pelvic lymph nodes (7 lymph nodes on the left and 3 on the right side) were all negative for tumor involvement. Immunohistochemical examination revealed positive staining for PSA, PSMA, CK7 (weakly positive), Ki-67 (2\%), tumor protein $\mathrm{p} 53$, human epidermal growth factor receptor 2 (HER2), AR, epidermal growth factor receptor (EGFR), multiple drug resistance (MDR), CD34, D2-40 and S-100, whereas the tumor was negative for CEA, estrogen receptor, progesterone receptor, p63 and B-cell lymphoma 2 (Fig. 3). Postoperatively, the patient received ADT with bicalutamide $150 \mathrm{mg}$ per day and goserelin $3.6 \mathrm{mg}$ every 4 weeks for 1.5 years; he was then followed up for 3 years and exhibited no signs of tumor recurrence or metastasis during that time. On the most recent follow-up in January 2018, the serum PSA level was $<0.003 \mathrm{ng} / \mathrm{ml}$ and the serum testosterone level was $<0.1 \mathrm{ng} / \mathrm{dl}$. The patient has a follow-up every 6 months with no further treatment at present.

\section{Discussion}

The incidence of prostate cancer diagnosis following TURP ranged between 8 and $18 \%$ prior to the introduction of PSA screening, and decreased to $8 \%$ thereafter (4). Mucinous carcinoma in TURP specimens is extremely rare and, to the best of our knowledge, has not been previously reported. We herein report two cases of mucinous carcinoma of the prostate diagnosed following TURP, with totally different clinical courses.

Mucinous carcinoma of the prostate is distinctly different from the secondary prostatic involvement by mucinous adenocarcinomas arising elsewhere (usually the rectum or bladder). The presence of typical acinar prostatic adenocarcinoma morphology and the cytological characteristics of the tumor often distinguish it from other malignancies in the majority of the cases (5). However, for less well-differentiated or undifferentiated tumors, the identification of their primary origin may 
A

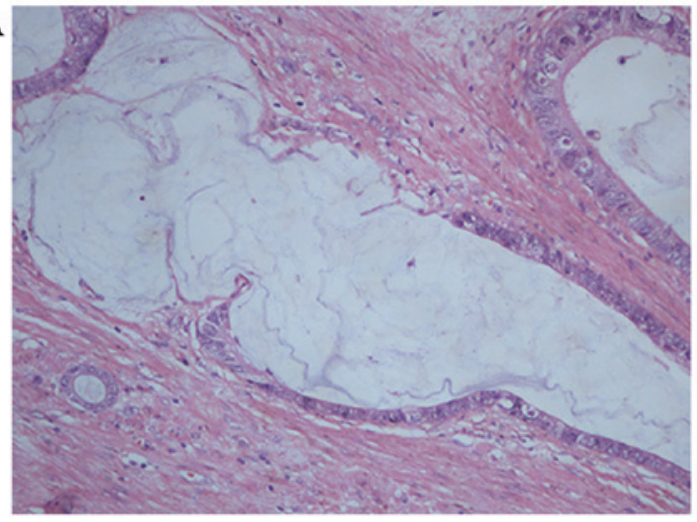

C

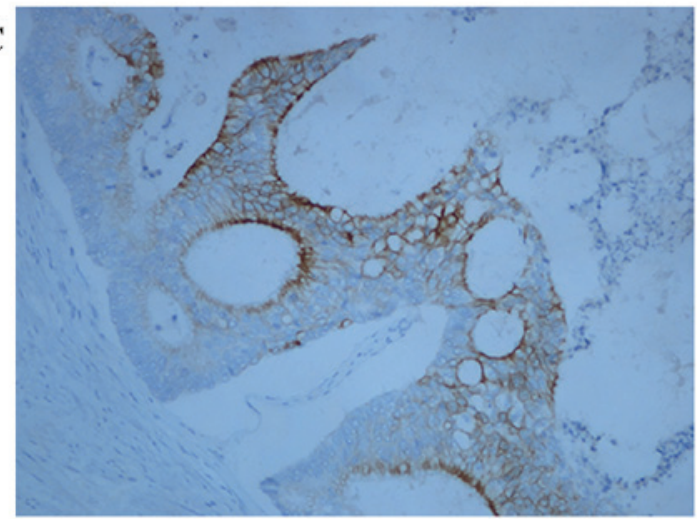

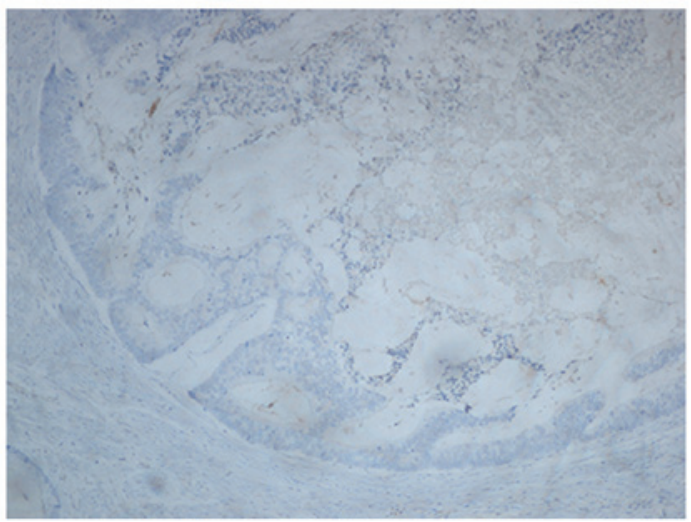

D

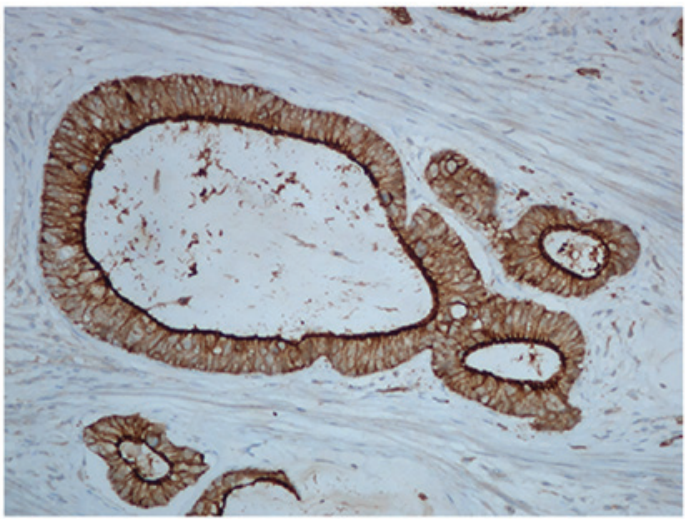

Figure 2. Microscopic characteristics of the surgical specimen in Case 1. (A) Hematoxylin and eosin staining; (B) negative staining for PSA; (C) positive staining for CK20; (D) positive staining for CEA (magnification, x200). PSA, prostate-specific antigen; CK, cytokeratin; CEA, carcinoembryonic antigen.

A

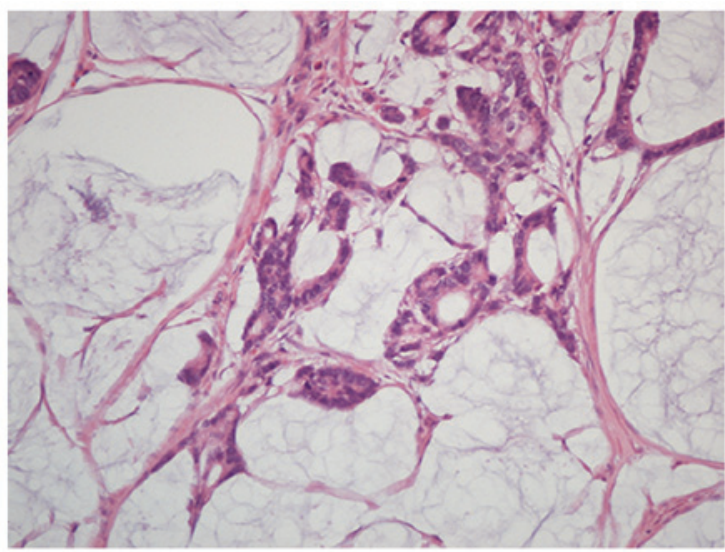

C

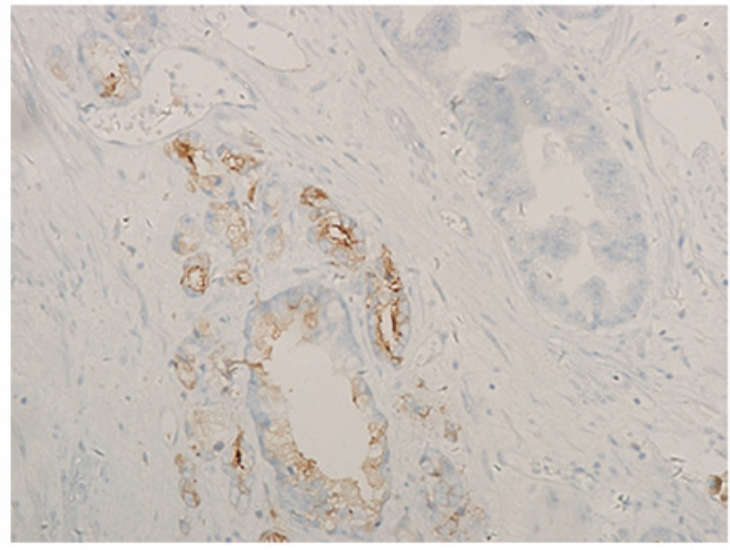

B

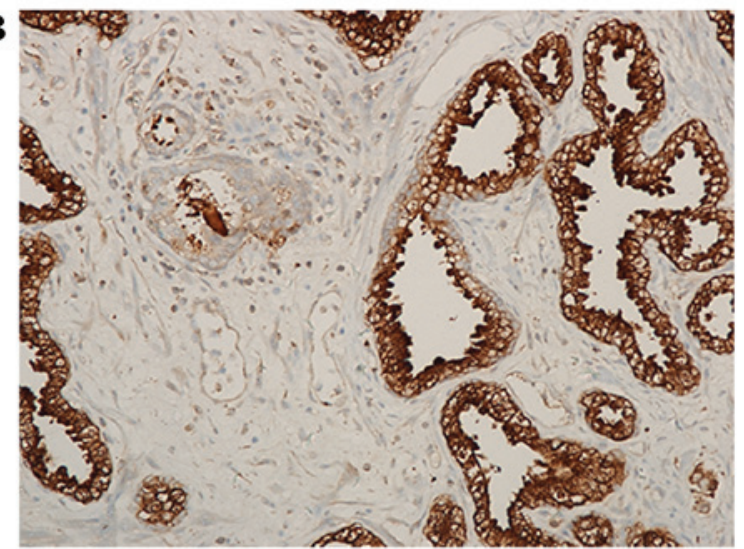

D

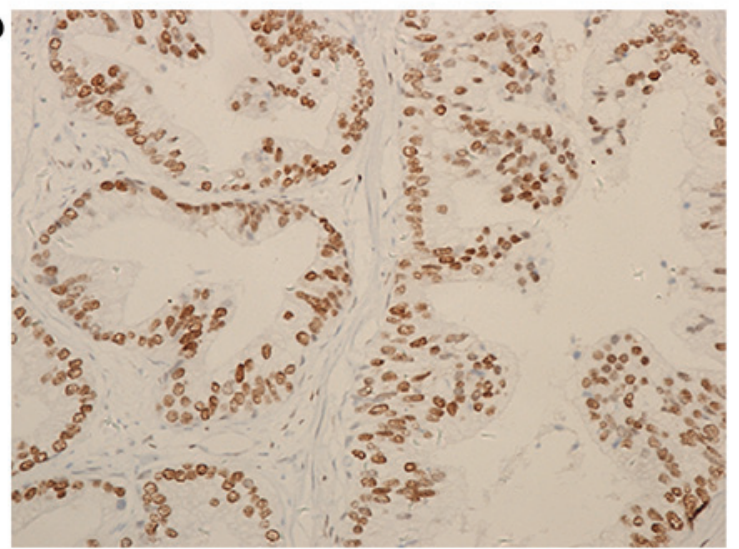

Figure 3. Microscopic characteristicsof the surgical specimen in Case 2. (A) Hematoxylin and eosin staining revealed the presence of large amounts of mucin; (B) strongly positive staining for PSA in the tumor cells; (C) weak and focal positive staining for PSMA; (D) positive staining for AR in the nuclei of the tumor cells (magnification, $\mathrm{x} 200$ ). PSA, prostate-specific antigen; PSMA, prostate-specific membrane antigen; AR, androgen receptor. 
be difficult. It has been proposed that negative staining for PSA in the tumor cells may help differentiate between primary and secondary tumors; however, PSA staining in undifferentiated prostatic cancer may also be negative (6), which makes this criterion unreliable. A number of alternative tumor markers have also been used in an attempt to distinguish the origin of the mucinous cancer of the prostate. Bassily et al (7) reported that the double-positive staining for CK7 and CK20 in the tumor cells may help distinguish urothelial from prostatic cancer; however, single-positive or double-negative staining for these two markers is of limited value. Furuta et al (8) reviewed 32 cases of mucinous adenocarcinoma of the prostate, and concluded that there were two subtypes: One that stains positive for PSA, which is a subtype of conventional adenocarcinoma, and another that stains positive for CEA and negative for PSA, which is derived from intestinal metaplasia with atypia of the prostatic urethra. In the present study, the surgical specimen from the first patient was negative for PSA and CK7, but positive for CK20 and CEA, and was confirmed to be a primary prostatic adenocarcinoma with bladder metastasis. However, in the second case, which was confirmed to be an organ-confined adenocarcinoma, the tumor stained positive for PSA and CK7, in addition to several other tumor markers, including Ki-67, p53, HER2, AR, EGFR and MDR. In the present study, PSA and CK7 staining alone are not considered reliable for the identification of the origin of mucinous adenocarcinoma of the prostate.

It was previously reported that the PSA levels in mucinous adenocarcinoma are comparable to those in non-mucinous tumor types $(1,3)$. However, in the present study, the PSA levels were substantially lower in patients with mucinous carcinoma: In the first case, a cT4 prostate cancer with bladder metastasis, the serum total PSA levels were $4.2 \mathrm{ng} / \mathrm{ml}$, whereas in the second case, a pT2 prostate cancer, the PSA levels were $0.75 \mathrm{ng} / \mathrm{ml}$. Taking into consideration the fact that mucinous carcinoma comprised $25 \%$ of the tumor mass in the first case and $100 \%$ in the second case, it may be hypothesized that a higher percentage of mucinous content in the tumor may be associated with a lower level of serum PSA. In clinical practice, an elevated PSA level is currently one of the major indicators for a prostate biopsy. Therefore, a normal or only slight increase in PSA levels in patients with mucinous carcinoma may result in omitting biopsy, thereby not diagnosing the tumor at an early stage.

The prognosis of mucinous carcinoma of the prostate remains controversial. It has been reported that the characteristics of mucinous adenocarcinoma of the prostate do not differ significantly from those of normal acinar adenocarcinoma in the clinical setting (9). The two types of tumors display similar biological behaviors, including PSA production and a propensity to develop bone metastases with advanced disease. In a series of 47 cases, the prognosis for mucinous adenocarcinoma of the prostate treated by radical prostatectomy is similar or better compared with nonmucinous adenocarcinoma (10). However, a more aggressive clinical course and a worse prognosis have also been reported in other previous studies $(10,11)$. In the present study, the two patients exhibited low levels of PSA during the course of ADT, which indicated a hormone responsiveness similar to conventional prostate adenocarcinoma; whereas worse outcome, in terms of recurrence and aggression, was associated with a higher Gleason score and positive surgical margins. In the organ-confined prostate cancer, mucinous adenocarcinoma treated by radical prostatectomy is potentially less aggressive compared with non-mucinous adenocarcinoma. However, routine adjuvant ADT may be problematic, as patients with mucinous adenocarcinoma of the prostate may have a variable outcome in terms of hormone responsiveness and recurrence (2). A negative expression of PSA in the surgical specimens may also predict a poor response to ADT; however, this hypothesis requires further confirmation through a well-designed prospective study with a larger patient population.

In conclusion, the clinical progression of mucinous adenocarcinoma of the prostate following TURP is similar to that of acinar prostate adenocarcinoma, depending on the Gleason score, focal involvement and surgical margins. The quantity of mucin present in the prostatectomy specimen appears to be inversely correlated with PSA levels, which may result in a delayed prostatic biopsy and diagnosis of this disease. Absence of PSA staining in the surgical specimen may be associated with poor response to ADT.

\section{Acknowledgements}

The authors would like to thank the staff of the Departments of Urology and Pathology at Zhongda Hospital for the technical support of this study.

\section{Funding}

No funding was received.

\section{Availability of data and materials}

Not applicable.

\section{Authors' contributions}

QF and MC designed the study. LijZ and LihZ collected the data. QF wrote the manuscript and approved the final version of the manuscript to be published.

\section{Ethics approval and consent to participate}

All procedures performed were in accordance with the ethical standards of the authors' Institutional Research Committee.

\section{Patient consent for publication}

Informed consent was obtained from the patients involved in the study regarding the publication of the case details and associated images.

\section{Competing interests}

All authors declare that they have no competing interests regarding the design or conduct of the present study.

\section{References}

1. Epstein JI and Lieberman PH: Mucinous adenocarcinoma of the prostate gland. Am J Surg Pathol 9: 299-308, 1985. 
2. Osunkoya AO and Epstein JI: Primary mucin-producing urothelial-type adenocarcinoma of prostate: Report of 15 cases. Am J Surg Pathol 31: 1323-1329, 2007.

3. Ro JY, Grignon DJ, Ayala AG, Fernandez PL, Ordonez NG and Wishnow KI: Mucinous adenocarcinoma of the prostate: Histochemical and immunohistochemical studies. Hum Pathol 21: 593-600, 1990.

4. Tombal B, De Visccher L, Cosyns JP, Lorge F, Opsomer R, Wese FX and Van Cangh PJ: Assessing the risk of unsuspected prostate cancer in patients with benign prostatic hypertrophy: A 13-year retrospective study of the incidence and natural history of T1a-T1b prostate cancers. BJU Int 84: 1015-1020, 1999.

5. Tran TA, Jennings TA, Ross JS and Nazeer T: Pseudomyxoma ovariilike posttherapeutic alteration in prostatic adenocarcinoma: A distinctive pattern in patients receiving neoadjuvant androgen ablation therapy. Am J Surg Pathol 22: 347-354, 1998.

6. Helpap B and Köllermann J: Undifferentiated carcinoma of the prostate with small cell features: Immunohistochemical subtyping and reflections on histogenesis. Virchows Arch 434: 385-391, 1999.

7. Bassily NH, Vallorosi CJ, Akdas G, Montie JE and Rubin MA: Coordinate expression of cytokeratins 7 and 20 in prostate adenocarcinoma and bladder urothelial carcinoma. Am J Clin Pathol 113: 383-388, 2000.
8. Furuta A, Naruoka T, Hasegawa N, Suzuki Y, Ikemoto I and Oishi Y: Mucinous adenocarcinoma of the prostate: A case report and review of 32 cases on immunohistochemical study of both PSA and CEA. Nippon Hinyokika Gakkai Zasshi 94: 570-573, 2003 (In Chinese).

9. Rhee AC, Olgac S, Ohori M and Russo P: Mucinous adenocarcinoma of the prostate: A case report of long-term disease-free survival and a review of the literature. Urology 63: 779-780, 2004.

10. Osunkoya AO, Nielsen ME and Epstein JI: Prognosis of mucinous adenocarcinoma of the prostate treated by radical prostatectomy: A study of 47 cases. Am J Surg Pathol 32: 468-472, 2008.

11. Manne RK and Haddad FS: Mucinous adenocarcinoma of prostate. Urology 33: 247-249, 1989. 